hep-th/9606122, HUTP-96/A025, IASSNS 96/60

\title{
Constraints on Low-Dimensional String Compactifications
}

\author{
S. Sethi* and C. Vafa \\ Lyman Laboratory of Physics \\ Harvard University \\ Cambridge, MA 02138, USA \\ and \\ E. Witten ${ }^{\dagger}$ \\ School of Natural Sciences \\ Institute for Advanced Study \\ Olden Lane, Princeton, NJ 08540, USA
}

\begin{abstract}
We study the restrictions imposed by cancellation of the tadpoles for two, three, and four-form gauge fields in string theory, M-theory and F-theory compactified to two, three and four dimensions, respectively. For a large class of supersymmetric vacua, turning on a sufficient number of strings, membranes and three-branes, respectively, can cancel the tadpoles, and preserve supersymmetry. However, there are cases where the tadpole cannot be removed in this way, either because the tadpole is fractional, or because of its sign. For M-theory and F-theory compactifications, we also explore the relation of the membranes and three-branes to the nonperturbative space-time superpotential.
\end{abstract}

$6 / 96$

* Supported in part by the Fannie and John Hertz Foundation (sethi@string.harvard.edu).

$\dagger$ Supported in part by NSF Grant PHY95-13835. 


\section{Introduction}

String theory compactifications below four dimensions have not been explored very thoroughly. This is partly because compactifications below four dimensions do not at first sight appear relevant to the observed four-dimensional universe. However, the picture has changed with recent developments [1] that show that certain four-dimensional compactifications of F-theory are related to Type IIA and M-theory compactifications below four dimensions. Compactifications below four dimensions may also be relevant to certain four-dimensional questions such as the vanishing of the cosmological constant [2]. Finally, given that the string world-sheet is itself two-dimensional, one naturally expects that some new interesting phenomena should occur upon compactification of string theory to two dimensions, or likewise of M-theory to three dimensions, or of F-theory to four dimensions.

In this paper, we begin to explore some aspects of these compactifications. In testing one of the predictions of string-string duality, it was shown in [3] that there is a one-loop interaction in Type IIA superstring theory of the form,

$$
\delta S=-\int B \wedge X_{8}(R),
$$

where $B$ is the NS-NS two-form of Type IIA which couples to the string, and $X_{8}(R)$ is an eight-form constructed as a quartic polynomial in the curvature. In particular, this implies that compactification on an eight-dimensional manifold $N$ with

$$
I=-\int_{N} X_{8} \neq 0,
$$

results in a one-point function for the $B$ field of the effective two-dimensional theory, destabilizing the vacuum. Given the relation between M-theory and Type IIA, this implies [4] that there is a similar term in the effective action of M-theory where $B$ is replaced by the three-form $C$ which couples to the membrane. The role of this term in M-theory has been discussed recently [7]. Similarly, given the relation between F-theory compactifications and M-theory [1], it follows that there is a similar term in the effective action of F-theory where $C$ is replaced by $A$, the four-form potential which couples to the F-theory threebrane. Note that since three-branes are the only branes in Type IIB invariant under $S L(2, \mathbf{Z})$, they play roughly the same role in F-theory that membranes play in M-theory, or strings in string theory $[8,9]$. 
To cancel the tadpoles present in these compactifications, we include in the vacuum $I$ strings, membranes, and three-branes in Type IIA, M-theory, and F-theory, respectively, where $I$ is defined in (1.2). The world-volumes of these strings, membranes, or threebranes are located at particular points on $N$, but stretch across the uncompactified space $\mathbf{R}^{n}$ (where $n=2,3$, or 4 in the three cases), thus preserving the $n$-dimensional Poincaré invariance. Some examples of this kind have been considered recently in [10,11]. There are two possible obstructions to canceling the tadpole in this way. One is that I may be fractional. Since branes only carry integer units of charge, there is no way to cancel the tadpole by including branes unless $I$ is an integer. A more subtle obstruction is that $I$ might be integral but negative. In this case, canceling the tadpole requires antibranes rather than branes. This does give a consistent tadpole-free vacuum, but without supersymmetry. The reason that anti-branes violate supersymmetry is that, first of all, compactification on the eight-manifold $N$ only preserves supersymmetries that have a definite eight-dimensional chirality (to be precise, some but not all supersymmetries of the preferred chirality are preserved in compactification on $N$ ). Likewise, inclusion of a brane or anti-brane that stretches across $\mathbf{R}^{n}$ at a particular point in $N$ preserves the supersymmetries of a definite eight-dimensional chirality. By definition, by "branes" we mean the objects that preserve the supersymmetries of the same chirality as those preserved in compactification on $N$; inclusion of anti-branes in compactification on $N$ therefore completely breaks the supersymmetry. To summarize, then, the tadpoles can be canceled in a supersymmetric fashion by inclusion of branes if and only if $I$ is a positive integer.

For Type IIA compactifications on a Calabi-Yau four-manifold $X, I$ is proportional to the topological Euler characteristic, $\chi$, of $N$. To see this, note that in the computation performed in [3], one ended up integrating the elliptic genus, defined by taking the odd spin structure for left-movers and the sum of all even spin structures for the right-movers, over the moduli space of elliptic curves. For compactifications with space-time supersymmetry, the sum over all even spin structures gives the same result as the odd spin structure. Thus, the net effect is to get a term proportional to the path integral with the odd spin structure for both left- and right-movers, which equals the world-sheet supersymmetric index $\operatorname{Tr}(-1)^{F}$ for this theory; for geometrical vacua, this is given by the Euler characteristic $\chi$ of $N$. The proportionality constant was fixed in [3], and is such that

$$
I=\frac{\chi}{24} .
$$


In the following section, we derive some topological constraints on the values of $\chi / 24$ for compactifications on Calabi-Yau four-folds. We shall also explore some examples of Type IIA compactifications where $\chi$ is negative. The case of $\mathrm{K} 3 \times \mathrm{K} 3$ is considered as an example of an F-theory compactification with $\mathrm{N}=2$ supersymmetry requiring twentyfour branes, but also admitting a dual realization as an F-theory compactification on the product of a Calabi-Yau three-fold with a $\mathbf{T}^{2}$. We conclude in section three with a discussion of the relation of these branes to the non-perturbatively generated space-time superpotential.

\section{Topological Constraints}

\subsection{Calabi-Yau Four-fold Compactifications}

We begin by considering a general Calabi-Yau four-fold, $N$. On such a space, we can compactify Type IIA, M-theory, or (if $N$ is elliptically fibered) F-theory to two, three, or four dimensions. While the formula $I=\chi / 24$ makes it appear that the obstruction to cancelling the anomaly by turning on branes resides generally in $\mathbf{Z}_{24}$, in actuality we will show that the Euler characteristic of a Calabi-Yau four-fold is always divisible by six, so the obstruction takes values in $\mathbf{Z}_{4}$.

To show that the Euler characteristic is always divisible by six, we will use index theory. The index of the Dolbeault operator $\bar{\partial}_{E_{p}}$ acting on anti-holomorphic forms with values in the bundle $E_{p}$ of holomorphic $(p, 0)$-forms is given by

$$
\operatorname{Ind}\left(\bar{\partial}_{E_{p}}\right)=\sum_{q=0}^{n=4}(-1)^{q} h^{p, q},
$$

where $h^{p, q}$ denotes the dimension of the cohomology group $H^{p, q}(N)$. This index is the twisted arithmetic genus, which can be expressed in terms of characteristic classes using the Atiyah-Singer index theorem:

$$
\operatorname{Ind}\left(\bar{\partial}_{E_{p}}\right)=\int_{N} \operatorname{Td}(N) \operatorname{ch}\left(\Omega^{p, 0}\right),
$$

where $\Omega^{p, q}$ is the bundle of complex-valued $(p, q)$-forms on $N$, Td is the Todd class, and ch is the Chern character. Since $c_{1}=0$, all toplogical indices for the four-fold can be expressed in terms of the Chern classes $c_{4}$ and $c_{2}^{2}$, where $\int_{N} c_{4}=\chi$ is the Euler characteristic. We 
begin by considering the untwisted arithmetic genus, corresponding to $p=0$ in (2.1). The Todd genus for a Calabi-Yau four-fold is given by,

$$
\operatorname{Td}(N)=\frac{1}{720}\left(3 c_{2}^{2}-c_{4}\right)
$$

This must be two for a Calabi-Yau four-fold, and therefore,

$$
\int_{N} c_{2}^{2}=480+\frac{\chi}{3}
$$

To complete the argument, we only have to consider the case $p=1$. The index in this case is given by

$$
\operatorname{Ind}\left(\bar{\partial}_{E_{1}}\right)=\frac{1}{180} \int_{N}\left(3 c_{2}^{2}-31 c_{4}\right)=8-\frac{\chi}{6} .
$$

The integrality of this index thus implies that $\chi$ is divisible by 6 . In the remaining case with $p=2$, the index,

$$
\operatorname{Ind}\left(\bar{\partial}_{E_{2}}\right)=\frac{1}{120} \int_{N}\left(3 c_{2}^{2}+79 c_{4}\right)=12+\frac{2}{3} \chi
$$

while further constraining the Hodge numbers, does not provide any new information about the divisibility of $\chi$.

Can we hope for a stronger restriction? It is easy to construct an example that shows that the Euler characteristic of a Calabi-Yau four-fold, while divisible by six, need not be divisible by 12 . Consider a Calabi-Yau hypersurface in $\mathbf{P}^{5}$ defined by a homogeneous polynomial of degree six. A straightforward application of the adjunction formula gives an Euler characteristic of 2610 for this space, and so $I=\frac{2610}{24}=108 \frac{3}{4}$; therefore, the obstruction to cancelling the tadpole is non-trivial, and takes values in $\mathbf{Z}_{4}$. It is easy to construct other examples of spaces with anomalies that cannot be canceled in a simple way by turning on branes.

\subsection{The Elliptically-Fibered Case}

A particular class of Calabi-Yau four-folds $N$ is of special interest, namely, those admitting elliptic fibrations with a section. Saying that $N$ admits an elliptic fibration means simply that there is a holomorphic projection $\pi: N \rightarrow B$, whose generic fiber is an elliptic curve; here $B$ is a complex three-fold, which except in rather special cases has $c_{1}$ at least generically positive. 
Such $N$ 's can be used to compactify F-theory to four dimensions [1]. Equivalently, we can view such an F-theory compactification as a Type IIB compactification on $B$, with seven-branes, in which the modulus of the elliptic fibration describes the variation of the dilaton and axion fields along $B$. From that point of view, the class dual to $12 c_{1}(B)$ can be identified with the class where the 7 -brane lives [12]. We can now attempt to rewrite $I=\chi / 24$ in terms of properties of $B$ alone. This turns out to be possible if the elliptically fibered manifold over $B$ is non-singular.

Before entering into a precise computation, let us note that $I$ will vanish if $c_{1}(B)=0$. Indeed, in that case, $B$ is a Calabi-Yau three-fold, and the four-fold $N$ is just $N=B \times$ $\mathbf{T}^{2}$, whose Euler characteristic vanishes. For this reason, one should expect $\chi(N)$ to be proportional to $c_{1}(B)$. If one assumes that $\chi$ can be written in terms of Chern classes of $B$, so that

$$
I=\frac{1}{24} \chi(N)=\int_{B}\left(\alpha c_{1} c_{2}+\beta c_{1}^{3}\right)
$$

(we omit a $c_{3}$ term since it would not vanish when $c_{1}(B)=0$ ), then the coefficients can be determined by considering some simple examples. For $N=\mathrm{K} 3 \times \mathrm{K} 3$ we have $\chi(N) / 24=24$. In this case, the base is $B=\mathrm{K} 3 \times \mathbf{P}^{1}$, for which $\int c_{1}^{3}=0, \int c_{1} c_{2}=48$, so $\alpha=\frac{1}{2}$. For another example, by considering an elliptically fibered Calabi-Yau over $\mathbf{P}^{3}$, for which $\chi / 24=972$, one gets $\beta=15$. The formula for $I$ is thus

$$
I=\int_{B}\left(15 c_{1}^{3}+\frac{1}{2} c_{1} c_{2}\right) .
$$

Note further that if the holonomy of $N$ is $S U(4)$, rather than a subgroup, then by a standard vanishing theorem, there are no holomorphic differential forms on $N$ except a constant zero-form and the Calabi-Yau four-form. There must likewise be no non-constant holomorphic differentials on $B$ (as a holomorphic differential on $B$ would pull back to a holomorphic differential on $N$ ). The arithmetic genus of $B$ (that is, $\left.\sum_{i=0}^{3}(-1)^{i} \operatorname{dim} H^{i, 0}(B)\right)$ is therefore equal to one. But the arithmetic genus of a threefold is $\int_{B} c_{1} c_{2} / 24$, so for this class of $N$ 's we can write

$$
I=12+15 \int_{B} c_{1}^{3}
$$

Since the integral in (2.4) is a positive integer, we see that for this class of $N$ 's, granted that $I$ can be written in terms of Chern classes, $I$ is always a positive integer, so that supersymmetric tadpole cancellation is always possible. 
It remains, then, to verify that in fact $I$ can be expressed in terms of Chern classes; in the process, we will recover the coefficients found above. This will be done by a standard computation of Chern classes. (Much of what is needed below can be found in chapter four of [13].) We recall that the elliptic four-fold $N$ with section can be described by a Weierstrass equation, which in terms of homogeneous coordinates $X, Y, Z$ reads $s=0$ where

$$
s=Z Y^{2}-X^{3}+a X Z^{2}-b Z^{3} .
$$

One can think of $X, Y$, and $Z$ as homogeneous coordinates on a $\mathbf{P}^{2}$ bundle $W \rightarrow B$. On the total space of this bundle is a line bundle that we will call $\mathcal{O}(1)$ (whose sections, when restricted to any $\mathbf{P}^{2}$ fiber of $W$, are functions of $X, Y, Z$ homogeneous of degree one). $Z$ is a section of $\mathcal{O}(1), X$ is a section of $\mathcal{O}(1) \otimes K^{-2}$, and $Y$ is a section of $\mathcal{O}(1) \otimes K^{-3}$, where $K$ is the canonical bundle of $B$, pulled back to $N$. ( $a$ and $b$ are sections of $K^{-4}$ and $K^{-6}$.) We write the total Chern class of $B$ as

$$
C=1+c_{1}+c_{2}+c_{3}
$$

where in particular $c_{1}=-c_{1}(K)$, and we set $\alpha=c_{1}(\mathcal{O}(1))$. The cohomology ring of $W$ is generated, over the cohomology ring of $B$, by the element $\alpha$ with the relation

$$
\alpha\left(\alpha+2 c_{1}\right)\left(\alpha+3 c_{1}\right)=0
$$

This relation expresses the fact that the homogeneous coordinates $Z, X$, and $Y$ have the following two properties: (i) they are sections respectively of the line bundles $\mathcal{O}(1), \mathcal{O}(1) \otimes$ $K^{-2}$, and $\mathcal{O}(1) \otimes K^{-3}$, whose first Chern classes are $\alpha, \alpha+2 c_{1}$, and $\alpha+3 c_{1}$; (ii) they have no common zeroes, so that the product of these first Chern classes vanishes.

The relation (2.7) holds in the cohomology ring of $W$. Our Calabi-Yau four-fold $N$ is defined by the equation $s=0$ where $s$, defined in $(2.5)$, is a section of $\mathcal{O}(1)^{3} \otimes K^{-6}$, which is a line bundle whose first Chern class is $3 \alpha+6 c_{1}=3\left(\alpha+2 c_{1}\right)$. Any cohomology class on $N$ that can be extended over $W$ (and this includes all those that we will require) can be integrated over $N$ by multiplying it by $3\left(\alpha+2 c_{1}\right)$ and then integrating over $W$. Since we are in that sense planning to multiply by $\alpha+2 c_{1}$, the relation $(2.7)$, in the cohomology ring of $N$, can be simplified to

$$
\alpha\left(\alpha+3 c_{1}\right)=0
$$

Now we wish to compute the Euler characteristic of $N$, or equivalently its fourth Chern class. To do this we use the adjunction formula, which says that the total Chern 
class of the projective space bundle $W$ is $C \cdot(1+\alpha)\left(1+\alpha+2 c_{1}\right)\left(1+\alpha+3 c_{1}\right)$, while the total Chern class $\bar{C}$ of $N$ is obtained by dividing this by $\left(1+3 \alpha+6 c_{1}\right)$, and is therefore

$$
\bar{C}=\left(1+c_{1}+c_{2}+c_{3}\right) \frac{(1+\alpha)\left(1+\alpha+2 c_{1}\right)\left(1+\alpha+3 c_{1}\right)}{\left(1+3 \alpha+6 c_{1}\right)} .
$$

The fourth Chern class of $N$, which we will call $\bar{c}_{4}$, is simply the quartic term in the expansion of (2.9). To compute it, we simply expand $\bar{C}$, dropping terms such as $c_{1}^{4}$ or $c_{1}^{2} c_{2}$, which vanish because $B$ is only three-dimensional, and setting $\alpha^{2}=-3 \alpha c_{1}$ according to (2.8). The result is

$$
\bar{c}_{4}=4 \alpha c_{1}\left(c_{2}+30 c_{1}^{2}\right) .
$$

The Euler characteristic of $N$ is

$$
\chi=\int_{N} \bar{c}_{4} .
$$

Integration over $N$ can be accomplished by first integrating over the fibers of $N \rightarrow B$, and then integrating over $B$. In the first step, one uses the fact that if $F$ is a generic fiber, then $\int_{F} \alpha=3$. The "3" reflects the fact that the equation $s=0$ is cubic in the homogenous coordinates $X, Y, Z$, so that the equation $Z=0$ (which is dual to the cohomology class $\alpha$ ) defines three points on $F$. So we get

$$
\chi=12 \int_{B} c_{1}\left(c_{2}+30 c_{1}^{2}\right) .
$$

Setting $I=\chi / 24$, this is equivalent to the result stated earlier in (2.3).

Before leaving this subject, let us note that the existence of a formula of this sort reflects the fact that on a D-brane world-volume, gravitational and gauge fields induce lower D-brane charges [14, 15]. In particular, it was shown in [14, 15] that if a $p$-brane world-volume $K$ has a non-zero first Pontryagin class $p_{1}$, there is an induced $p$-4-brane charge given by $\frac{1}{48} p_{1}(K)$; if $K$ is a complex manifold this is the same as $c_{2}(K) / 24$. In the case at hand, the seven-brane world-volume is given by $\mathbf{R}^{4} \times K$ where $K$ (the discriminant of $N \rightarrow B$ ) is a complex surface in $B$ whose cohomology class is $12 c_{1}$. The induced $3-$ brane charge is thus roughly $\int_{B}\left(12 c_{1}\right)\left(c_{2}(K) / 24\right)$. One can then, roughly, express $c_{2}(K)$ as a linear combination of $c_{1}(B)^{2}$ and $c_{2}(B)$, obtaining for $\chi$ a formula along the lines above. The difficulty in making this approach precise is that for any elliptic four-fold $N$ of $S U(4)$ holonomy, $B$ has singularities (corresponding to points at which the fiber of $N \rightarrow B$ has a cusp, that is points at which $a=b=0$ in (2.5)), and care is required to determine the 
contribution of these singularities to the three-brane charge. The approach we gave above, in which everything is referred to the $\mathbf{P}^{2}$ bundle $W$, sidesteps such issues.

The above computation of the Euler characteristic is valid precisely for elliptic fourfolds with (i) a section, and (ii) a smooth Weierstrass model. If either condition is relaxed, the formula does not hold. In particular, to analyze compactifications of $F$-theory with generic non-trivial unbroken gauge groups, one would like to understand what happens for elliptic four-folds with a section whose Weierstrass model is not smooth. We will not try to analyze that case.

\subsection{Examples with $\chi<0$.}

We have already given examples in which tadpole cancellation fails because $I$ is not integral. We here wish to examine the other possible obstruction, which is that $I$ might be negative.

Constructing examples of Calabi-Yau four-folds with $\chi<0$ is difficult, essentially because $h^{2,1}$, which is the only non-vanishing Hodge number contributing to $\chi$ with a negative sign, is usually small in simple examples. We wonder whether some of the usual constructions of Calabi-Yau's as complete intersections in projective spaces or even in more general toric varieties may always give examples with $\chi>0$.

If one is willing to consider more general conformal field theories such as orbifolds with discrete torsion ([16]; see [17] for a recent discussion and geometrical interpretation of some examples), then it is not difficult, at least in the context of Type IIA compactification from ten to two dimensions, to find examples for which the number $I$ of branes needed to cancel the tadpole is negative. These are thus superficially reasonable Type IIA compactifications in which supersymmetry is spoiled by the wrong sign of the tadpole. It is plausible that these examples also have analogs in M-theory and F-theory.

We will consider a simple example for illustration. This example has a non-zero $h^{2,0}$, and so gives $N=4$ supersymmetry in $d=2$. 1

The example is a toroidal orbifold, the target space being $\mathbf{T}^{8} / \mathbf{Z}_{2} \times \mathbf{Z}_{2}$. We describe $\mathbf{T}^{8}$ via four complex coordinates $z_{i}$. The generator, $\alpha$, of the first $\mathbf{Z}_{2}$ acts on the coordinates by, $z_{i} \rightarrow-z_{i}$, for $i=1,2$. The generator for the second $\mathbf{Z}_{2}$, denoted $\beta$, acts similarly

1 As is customary, we define the Hodge numbers $h^{p, q}$ of a two-dimensional $N=2$ superconformal field theory in terms of the number of Ramond ground states with given $U(1)_{L} \times U(1)_{R}$ quantum numbers. 
on the coordinates $z_{3}, z_{4}$. Without discrete torsion, this model is just $\mathrm{K} 3 \times \mathrm{K} 3$ at a point in its moduli space with orbifold symmetry. With discrete torsion, the situation changes. In this model, the choice of a non-trivial discrete torsion is unique. Specifying the phase, $\epsilon(\alpha, \beta)=-1$, for a genus one path integral with $\alpha$ and $\beta$ twists, determines the weights for all other path integral sectors. Let us compute the Hodge diamond with this discrete torsion. The contribution from the untwisted sector is unchanged from the standard orbifold computation, and is given by,

$$
\left(\begin{array}{ccccc}
1 & 0 & 2 & 0 & 1 \\
0 & 8 & 0 & 8 & 0 \\
2 & 0 & 20 & 0 & 2 \\
0 & 8 & 0 & 8 & 0 \\
1 & 0 & 2 & 0 & 1
\end{array}\right)
$$

where the $(p, q)$ entry is $h^{p, q}$. The contribution from the three twisted sectors is more interesting. Let us consider the sector twisted by the element $\alpha \beta$ in the quotient group. The fixed point set consists of $4^{4}$ points, which, without discrete torsion, would contribute $4^{4}$ to $h^{2,2}$. The shift of the $(p, q)$ charges in a twisted sector is described in 18. Because $\epsilon(\alpha, \alpha \beta)=\epsilon(\beta, \alpha \beta)=-1$, the fixed points are no longer invariant under the action of the quotient group, and are projected out; hence there is no contribution from this sector. The remaining two fixed point sectors give equal contributions, so we need only consider the sector twisted by $\alpha$. The fixed point set is 16 copies of $\mathbf{T}^{4}$. Because of the discrete torsion, the only operators which survive projection have (unshifted) charges given by,

$$
\left(\begin{array}{ccc}
0 & 32 & 0 \\
32 & 0 & 32 \\
0 & 32 & 0
\end{array}\right) .
$$

The total contribution from twisted and untwisted sectors gives a Hodge diamond,

$$
\left(\begin{array}{ccccc}
1 & 0 & 2 & 0 & 1 \\
0 & 8 & 64 & 8 & 0 \\
2 & 64 & 20 & 64 & 2 \\
0 & 8 & 64 & 8 & 0 \\
1 & 0 & 2 & 0 & 1
\end{array}\right)
$$

For this space, $\chi$ is $-8(24)$, so we require 8 anti-branes to cancel the tadpole. Because $\chi$ is negative, this results in supersymmetry breaking. 


\subsection{F-Theory on $\mathrm{K} 3 \times \mathrm{K} 3$}

There are several ways to obtain $N=2$ supersymmetry in four dimensions from F-theory. One possibility is F-theory compactification on the product of a Calabi-Yau three-fold $C$ with a two-torus. If $C$ is elliptically fibered over a base $B$, there are two apparently distinct (but equivalent under further compactification) versions of F-theory on $C \times \mathbf{T}^{2}$ : one can consider Type IIB on $C$ itself or on $B \times \mathbf{T}^{2}$. We will be interested in the latter case. Another $N=2$ model in four dimensions is F-theory on $\mathrm{K} 3 \times \mathrm{K} 3$ where one of the K3's is elliptic (the latter compactification has been considered in [19]). It is natural to ask if the two theories can be dual to one another. We will now see that with a proper choice of three-fold $C$, this is indeed the case. In the analysis we will see that in this special case, the branes we have encountered in this paper are related to more familiar things, and we will get a check on the proportionality constant in the formula $I=\chi / 24$.

One starts from the duality of F-theory on $\mathrm{K} 3$ to heterotic strings on $\mathbf{T}^{2}$. By compactifying both sides on a K3 down to four dimensions, we get a duality between F-theory on $\mathrm{K} 3 \times \mathrm{K} 3$ with heterotic strings on $\mathrm{K} 3 \times \mathbf{T}^{2}$. We consider the case where we do not excite a gauge bundle over the $\mathrm{K} 3$ on the heterotic side, but cancel the heterotic world-sheet anomaly by turning on 24 five-branes (wrapped around $T^{2}$ ) [20]. The eight dimensional duality between F-theory and heterotic strings implies that we need to turn on 24 three-branes on the F-theory side. This is, of course, in agreement with the fact that for a $\mathrm{K} 3 \times \mathrm{K} 3$ compactification, we have $I=\chi / 24=24$. On the other hand, we can look at $\mathrm{K} 3 \times \mathbf{T}^{2}$ compactification of the heterotic string in another way: we fiber the eight-dimensional duality between the heterotic string on $\mathbf{T}^{2}$ and F-theory on K3, to obtain six-dimensional duality between heterotic strings on K3 and F-theory on a certain Calabi-Yau three-fold [12]. This duality can be extended to the case where the heterotic string has trivial gauge bundle (and 24 fivebranes) by an appropriate number of blowups on the F-theory side [21]. A description of the resulting Calabi-Yau three-fold $C$, which has $\left(h^{11}, h^{21}\right)=(43,43)$ has been given in [22]. We then compactify this further on a $\mathbf{T}^{2}$ to four dimensions. On the heterotic side of course we have the same compactification as above; we have simply exchanged the 9-10 coordinate with 5-6 coordinate. On the F-theory side, this geometric symmetry of the heterotic string translates to a duality between F-theory on $C \times \mathbf{T}^{2}-$ with this particular $C$ - and F-theory on $\mathrm{K} 3 \times \mathrm{K} 3$. 


\section{Non-Perturbative Superpotential}

In this paper we have been analyzing compactifications of F-theory to four dimensions (or related compactifications of Type IIA superstring theory or M-theory to two or three dimensions) on a Calabi-Yau four-fold $N$. In the limit that $N$ has large radius, this gives a four-dimensional vacuum with $N=1$ supersymmetry. Many of these models, however, generate non-perturbative superpotentials - exponentially small in the large radius limit - because of instantons that arise from five-brane wrapping [23]. This occurs when the $F$-theory base $B$ has a divisor $Q$ with certain properties; it suffices to have $H^{i, 0}(Q)=0$, $i=1,2$, and $H^{0}\left(Q, N_{Q}\right)=0, N_{Q}$ being the normal bundle to $Q$ in $B$. We wish in this concluding section to briefly consider a few issues involving the relation of these nonperturbative superpotentials to the branes that have been our main interest in this paper.

First of all, we note a rather surprising fact about the non-perturbative superpotentials. Given any $F$-theory base $B$ that does not have a divisor that can generate a superpotential, we can create a complex manifold $B^{\prime}$ that does have such a divisor by blowing up a point $P \in B$; we simply let $Q$ be the "exceptional divisor" created in the blow-up. If there is a Calabi-Yau four-fold $N^{\prime}$ over $B^{\prime}$ that can be used in F-theory compactification, then in compactification on $N^{\prime}$ there will be a non-perturbative superpotential.

Now, by analogy with what happens for F-theory compactification to six dimensions [12,24, one might expect that phase transitions, perhaps of an exotic nature, would occur between F-theory on $N \rightarrow B$ and F-theory on $N^{\prime} \rightarrow B^{\prime}$. The superpotential, however, may be identically zero for F-theory on $N$, while it is definitely non-zero for F-theory on $N^{\prime}$. Although there does not quite seem to be a contradiction in the fact that there is a superpotential in $N^{\prime}$ and none on $N$, we do find it challenging to try to reconcile this fact with the expectation that a physical transition from compactification on $N$ to compactification on $N^{\prime}$ is possible.

We will not meet this challenge here, but point out one fact which must be part of the story. The number of branes in compactification on $N^{\prime}$ is not the same as that in compactification on $N$. In fact, if $B^{\prime}$ is obtained from $B$ by blow-up of a point, then $\int_{B^{\prime}} c_{1}\left(B^{\prime}\right)^{3}-\int_{B} c_{1}(B)^{3}=-8$. Thus there are $8 \cdot 15=120$ fewer branes if the base is $B^{\prime}$ than if it is $B$. Presumably the interpretation is that a point $P \in B$ can be blown up only if 120 branes simultaneously arrive at $P$ and annihilate. There is no surprise per se in the fact that some parameters must be adjusted to make blow-up of a point in the base possible; this is also so in F-theory compactification to six dimensions [12,24], where the 
relevant parameters are complex structure modes of the elliptic three-fold that is fibered over $B$. The novelty is merely that among the parameters that must be so adjusted are the positions of branes.

Another obvious question raised by the presence of branes in these vacua is how the non-perturbative superpotential depends on the brane positions. We note that the nonperturbative superpotential generated by an instanton associated with a divisor $Q$ should be expected to get no zero or pole from the motion of branes in the vacuum as long as those remain disjoint from $Q$. However, a singular behavior as one of the vacuum branes approaches $Q$ might be expected, and would be well worth analyzing.

We would like to thank A. Klemm for valuable discussions. The research of SS and CV was supported in part by NSF grant PHY-92-18167. The research of EW was supported in part by NSF grant PHY-9513835. 


\section{References}

[1] C. Vafa, "Evidence For F Theory", hep-th/9602022.

[2] E. Witten, Mod. Phys. Lett. A10 (1995) 2153.

[3] C. Vafa and E. Witten, Nucl. Phys. B447 (1995) 261.

[4] M. Duff, J. Liu, and R. Minasian, Nucl. Phys. B452 (1995) 261.

[5] P. K. Townsend, Phys. Lett. B350 (1995) 184.

[6] E. Witten, Nucl. Phys. B443 (1995) 85.

[7] K. Becker and M. Becker, "M-Theory on Eight-Manifolds", hep-th/9605053.

[8] A. A. Tseytlin, "Self-Duality of Born-Infeld Action and Dirichlet 3-Brane of Type IIB Superstring Theory", hep-th/9602064.

[9] D. P. Jatkar and S. K. Rama, "F Theory From Dirichlet Three-Branes", hepth/9606009.

[10] E. Witten, "Five-Branes And $M$-Theory On An Orbifold," Nucl. Phys. B463 (1996) 383.

[11] A. Sen, "Duality and Orbifolds", hep-th/9604070.

[12] D. Morrison and C. Vafa, "Compactifications of F Theory on Calabi-Yau Threefolds. I,II", hep-th/9602114, hep-th/9603161.

[13] R. Bott and L. W. Tu, Differential Forms In Algebraic Topology (Springer-Verlag, 1982).

[14] M. Bershadsky, V. Sadov and C. Vafa, Nucl. Phys. B463 (1996) 398.

[15] M. Green, J. Harvey and G. Moore, "I-Brane Inflow and Anomalous Couplings on D-branes", hep-th/9605033.

[16] C. Vafa, Nucl. Phys. B273 (1986) 592.

[17] C. Vafa and E. Witten, J. Geom. Phys. 15 (1995) 189.

[18] E. Zaslow, Comm. Math. Phys. 156 (1993) 301.

[19] M. Douglas and M. Li, "D-brane Realization of Super Yang-Mills Theory in FourDimensions", hep-th/9604041.

[20] M.J. Duff, R. Minasian and E. Witten, "Evidence for Heterotic/Heterotic Duality", hep-th/9601036.

[21] N. Seiberg and E. Witten, "Comments on String Dynamics in Six-Dimensions", hepth/9603003.

[22] P. Candelas and A. Font, "Duality Between the Webs of Heterotic and Type II Vacua", hep-th/9603170.

[23] E. Witten, "Nonperturbative Superpotentials in String Theory", hep-th/9604030.

[24] E. Witten, "Phase Transitions In M-Theory And F-Theory," hep-th/9603150. 